\section{PCR-Based RNA Probes: A Quick and Sensitive Method to Improve Whole Mount Embryo In Situ Hybridizations}

BioTechniques 30:_____ (April 2001)

\begin{abstract}
We have developed a PCR-based technique for the preparation of RNA probes that can be used for whole mount in situ hybridization on embryos. T3 or T7 RNA polymerase promoters were introduced at the $5^{\prime}$ end of gene-specific oligonucleotide primers enabling direct in vitro transcription of purified PCR fragments. We show for various marker genes in Xenopus embryos
\end{abstract}

that this method provides equivalent results as compared to conventional vector-based probe preparation, even when fluorescence detection (FISH) is applied. This method offers a rapid and useful means to prepare gene-specific in situ probes predominantly for expression screens or detection of splice variants that previously required time-consuming cloning steps.

\section{INTRODUCTION}

Whole mount in situ hybridization has become one of the standard techniques in modern biosciences. It is often used for studying endogenous spatial and temporal expression patterns of novel isolated genes. It also serves as a pow erful tool in experiments when altered gene expression is examined upon genetic and epigenetic manipulations. Commonly, digoxigenin (DIG)-labeled cRNAs are used for whole mount in situ hybridizations, whereby the preparation of these probes involves an initial timeconsuming cloning step using a bacteriophage promoter-carrying vector. Specifically designed oligonucleotide primers containing T3 or T7 RNA polymerase promoters at their $5^{\prime}$ ends were successfully used by Logel et al. (7) to produce PCR fragments that could be directly used for in vitro transcription, omitting plasmid preparation steps. We applied this technique for labeling of whole mount in situ probes and compared the results with conventional vector-derived probes. We demonstrate that equal results are obtained for the expression of the marker genes Xbra, Xtwi, XneuroD, and Xcad-6 $(2,5,6,9)$ in Xenopus embryos. We show that the high sensitivity of the PCR probes also allows fluorescence signal detection. In com parison to conventional vector-based probe preparation, this PCR approach offers convenience and saves time. 


\section{Short Technical Reports}

\section{MATERIALS AND METHODS}

\section{Production of PCR-Derived Antisense RNA Probes}

PCR fragments representing base pairs 821-1493 of the Xbra coding region, base pairs $20-1012$ of the Xtwi coding region, base pairs 13-1042 of the $\mathrm{xNeuroD}$ coding region, and base pairs 868-1512 of the Xcad-6 coding region $(2,5,6,9)$ were produced under standard PCR conditions using Taq DNA polymerase (Eurogentec, Seraing, Belgium) (35 cycles and $57^{\circ} \mathrm{C}$ annealing temperature). Plasmid DNA (10 ng) containing the full-length cDNAs served as PCR templates. In each case, the gene-specific downstream primer contained an artificially introduced $\mathrm{T} 3$ promoter at its $5^{\prime}$ end to enable synthesis of antisense transcripts. In the case of Xcad-6, the upstream primer additionally contained a T7 promoter at its $5^{\prime}$ end for the synthesis of sense transcripts. PCR fragments were gel-purified using Quick-spin ${ }^{\mathrm{TM}}$ columns (Amersham Pharmacia Biotech, Piscataway, NJ, USA), and $50 \mathrm{ng}$ were subsequently used as template DNA for in vitro transcription incorporating Dig-UTP via a Dig-labeling kit (Roche Molecular Biochemicals, Mannheim, Germany). Primer sequences are shown below, whereby promoter sequences are underlined: Xbra (5'-GGATGAGGGAATCGATAGTC-3' and $5^{\prime}$-AATTAACCCTCACTAAAGG GCTCAGGCCCAGGAAATACTG-3'), Xtwi (5'-GACACACTGTGATAGGG CTG- $3^{\prime}$ and 5' $5^{\prime}$ AATTAACCCTCACTAAAGGGGTGGCAACGCATGCATGCAG-3'), xNeuroD (5'-GACCAAATCGTATGGAGAGAATGGG-3' and $5^{\prime}$-AATTAACCCTCACTAAAGGGGGCACTCATGACTCTTTCATGG-3'), and Xcad-6 (5'-TAATACGACTCACTATAGGGCCCTGTGGATTCTTCCA TT- $3^{\prime}$ and $5^{\prime}$-AATTAACCCTCACTAA AGGGACAAATATCTCATACAACA TG-3').

\section{Whole Mount In Situ Hybridization}

Whole mount in situ hybridization was performed as described by Hollemann and Pieler (4) for PCR and conventional plasmid-derived antisense probes where the transcripts were detected via phosphatase reaction. Plas- mid-derived probes in each of the three marker-genes corresponded to the fulllength gene bank sequences. Fluorescence detection of transcripts shown for the Xtwi PCR-derived probe was carried out essentially as described in http://www.people.virginia.edu/ lad4x/ fluor_insitu/protocol.html. Alterations were made concerning the following points: peroxidase inactivation was omitted after antibody washes, and the color substrate was diluted 1:50 in amplification diluent delivered with the $\mathrm{TS}^{\mathrm{TM}}$ kit $\left(\mathrm{NEN}^{\circledR}\right.$ Life Science Products, Boston, MA, USA). Additionally, the washing time after incubation with Red-FISH was extended to $48 \mathrm{~h}$.

\section{Whole Mount Immunofluorescence}

Whole mount staining against fibronectin was performed using mab 6D9 (1) according to standard proto- cols. Embryos subjected to antibody staining were post-fixed for $2 \mathrm{~h}$ in MEMFA (100 mM MOPS, pH 7.4, 2 $\mathrm{mM}$ EGTA, $1 \mathrm{mM} \mathrm{MgSO}_{4}$, $4 \%$ formaldehyde) after completion of the fluorescence in situ procedure described above. Subsequently, vibratome sections were prepared that enabled simultaneous chemiluminescent detection of transcripts and protein immunostaining using confocal laser microscopy.

\section{RESULTS AND DISCUSSION}

Figure 1A shows the gel-purified PCR products resulting from PCR using the primer pairs and conditions described above. Bands of the expected size were obtained for all three of the marker-genes (Xbra, Xtwi, and XneuroD), showing that the addition of the T3 promoter sequence to the

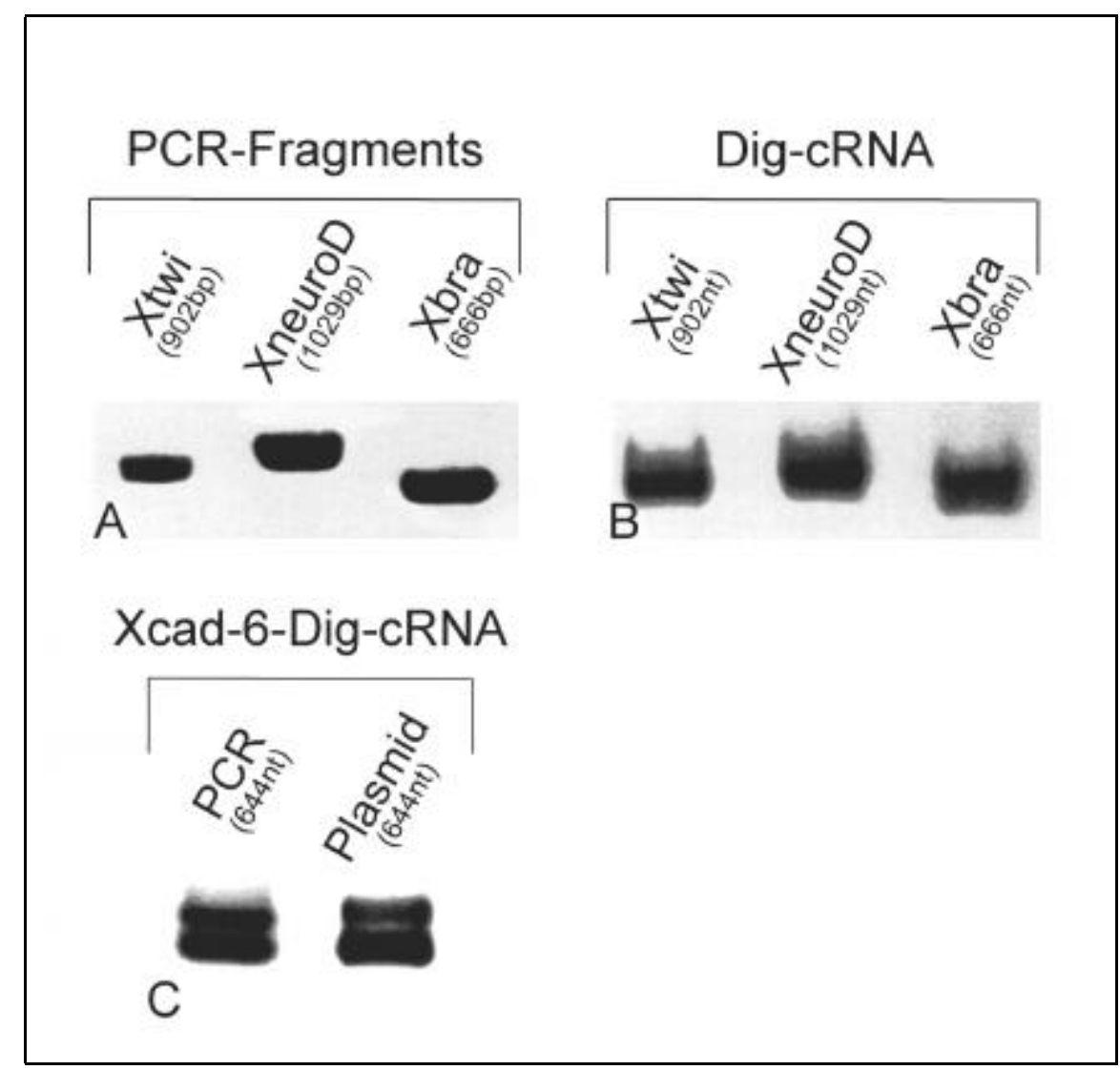

Figure 1. Gel-purified PCR-products (A) containing a T3 promoter artificially introduced via the downstream primer were used to produce DIG antisense RNAs (B) corresponding to Xbra, Xtwi, and XneuroD. The lengths of the PCR products are indicated. (C) Comparison of the transcription efficiencies using an identical 644-bp fragment of Xcad-6 either as a PCR product or contained in a linearized pBKS vector. Equivalent amounts of DIG antisense RNA were obtained using T3 RNA polymerase. As often observed under native gel conditions, the RNA forms double bands here. 


\section{Short Technical Reports}

primers did not impair the amplification of the DNA (Figure 1A). Thus, template DNAs were generated that could be directly used for in vitro transcription incorporating Dig-UTP in the transcripts. Aliquots of the corresponding samples are shown in Figure 1B.
The amount of the DIG-labeled transcripts generated from PCR templates was equivalent to those derived from a linearized plasmid. To demonstrate this, a 644-bp fragment of Xcad-6 was subcloned into $\mathrm{pBKS}$, and an antisense probe was transcribed from the lin-
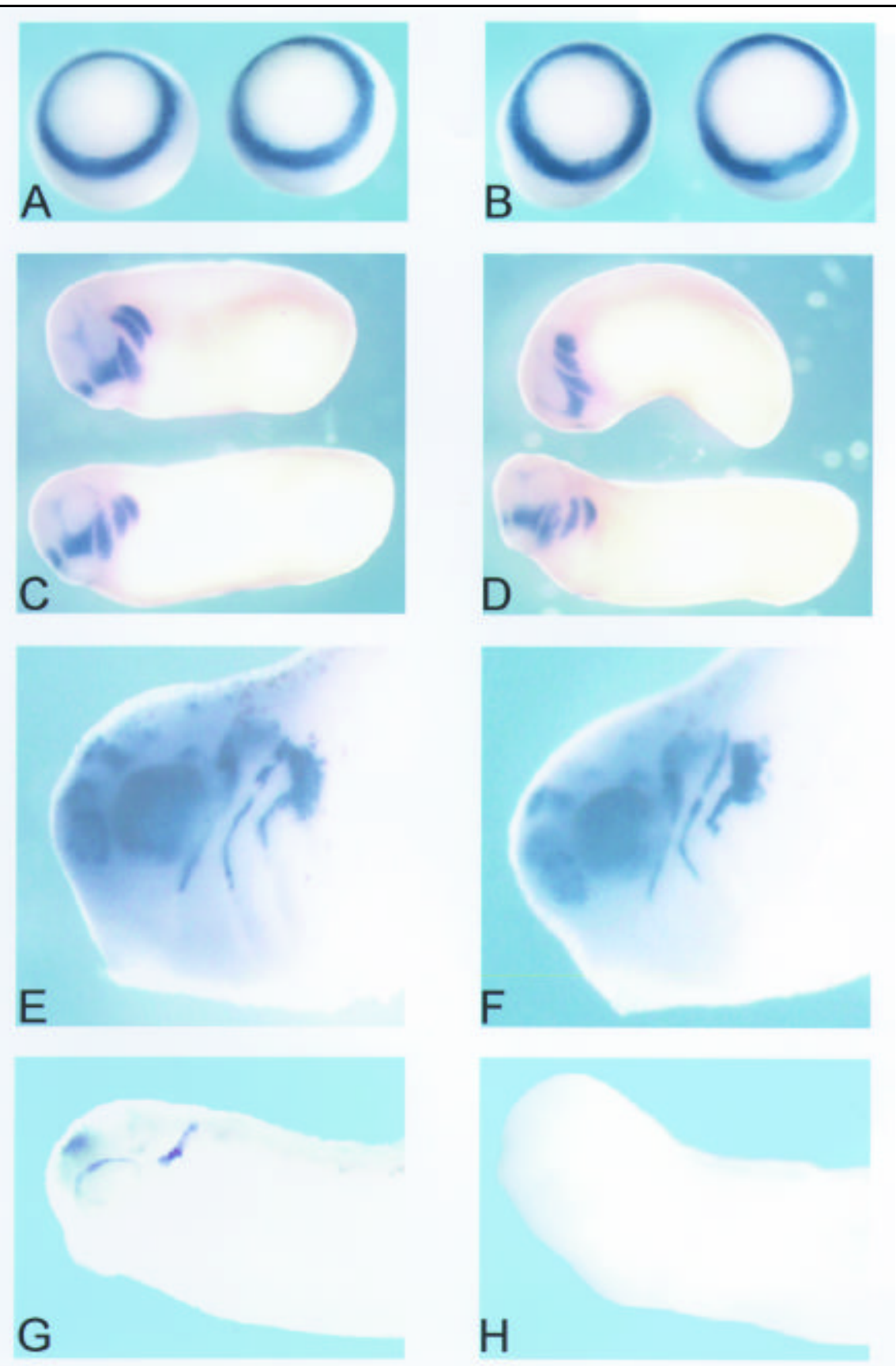

Figure 2. DIG-labeled antisense RNAs produced conventionally $(A, C$, and E) or via the PCR approach $(B, D$, and $F)$ were used for whole mount in situ hybridizations. Equal results were obtained for the marker genes Xbra (A and B), Xtwi (C and D), and XneuroD (E and F). The additional introduction of a T7 promoter in the upstream primer allows for production of antisense $(\mathrm{G})$ and sense $(\mathrm{H})$ probes from the same PCR template as shown for Xcad-6. Sense probes show no signal. earized plasmid. A corresponding 644bp PCR product was generated in parallel by introducing the $\mathrm{T} 3$ promoter in the downstream primer, thereby enabling transcription from the PCR product directly. As expected, either approach generated DIG RNA molecules of the same length and equivalent amounts (Figure 1C). Subsequent whole mount in situ hybridizations revealed Xcad-6 transcripts in the brain, the peripheral nervous system, and in neurogenic placodes (Figure 2G) (2). As for the marker genes described below, no differences between plasmid and PCR-derived probes were observed (data not shown). In Figure 2, whole mount in situ hybridizations of albino embryos using PCR- and conventional plasmid-derived probes were com pared. To demonstrate the application of this novel technique over a wide spectrum of developmental stages, various well-described markers were chosen so that gastrula-to-tailbud embryos were included. The early mesodermal marker Xbra (9) shows its typical ring

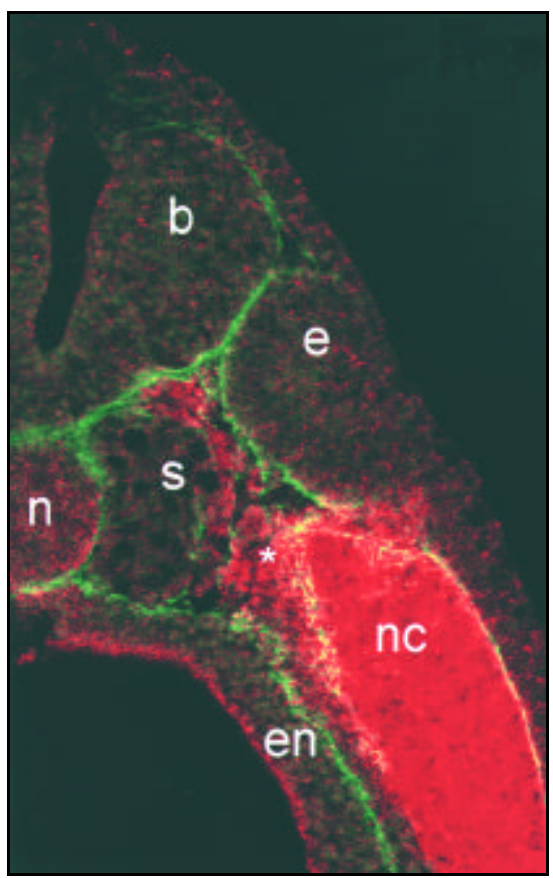

Figure 3. Fluorescence detection of Xtwi mRNA via PCR-derived antisense probe (red) in combination with antibody staining against fibronectin (green). b, brain; e, eye anlage; $s$, somite; n, notochord; en, endoderm; nc, Xtwi positive neural crest cells of the mandibular crest segment; *, subpopulation of single migratory Xtwi-positive cells. 
of expression around the blastopore using either the PCR- (Figure 2A) or the conventional plasmid-derived (Figure $2 B)$ probe. No alterations during the entire in situ procedure were necessary to obtain the same signal intensity, despite the much shorter length of the PCR template as compared to the fulllength cDNA and potential base exchanges due to Taq DNA polymerase. Equivalent results were also produced for the neural crest marker Xtwi (Figure 2C, PCR-derived probe; Figure 2D, plasmid-derived probe) (5) at early and late tailbud stage, as well as for the proneural marker XneuroD (Figure 2E, PCR-derived probe; Figure $2 \mathrm{~F}$, plasmid-derived probe) (6) that shows a highly specific pattern in brain and neurogenic placodes in late tailbud specimens (8). PCR-derived probes are also sensitive enough to allow fluorescence detection of mRNAs, giving the advantage of localizing transcripts and pro- teins in the same section when highquality microscopes are used. Figure 3 shows Xtwi in situ hybridization (red) in combination with antibody staining against fibronectin (green) as an example of this technique.

The novel technique of PCR-based in situ probes described here provides a useful tool in a number of aspects. First, many plasmids do not offer the appropriate bacteriophage promoters flanking the cloned cDNA. This is the case for many TA cloning vectors used for rapid cloning of PCR products and for twohybrid activation domain fusion vectors. Conventionally, the isolated cDNA in such screening vectors has to be subcloned into a standard vector to allow transcription of in situ probes. The selection of interesting clones can be dramatically accelerated by avoiding cloning and plasmid preparation steps using the PCR approach. Even crude DNA can be used as a template, as long as sufficient sequence information is available to design the appropriate PCR primer pair. A further application is the generation of highly specific probes from full-length clones, when the gene of interest belongs to a family of closely related members or when splice variants are found. Here, the PCR approach facilitates the generation of highly specific probes by amplifying the sequence regions of lowest similarity. However, the minimal length of the PCR fragment should be at least 300 bp because shorter probes are less sensitive. We have successfully applied the PCR-based approach to a number of cDNAs including our recently published Xenopus Cadherin-6 (Xcad-6) clones (2), Xenopus Reptin (3), and various novel clones isolated using the yeast two-hybrid methodology. Excellent results were also obtained when fluorescein-labeled probes were used for hybridization and when transcription was performed via a T7 promoter introduced in the forward primer. The latter approach enabled simultaneous synthesis of antisense and sense probes from the same PCR tem plate, which is shown for Xcad-6 (2). The antisense probe revealed Xcad-6 transcripts in the brain, the eye, the peripheral nervous system, and in neurogenic placodes (Figure 2G), and, as expected, no signal was observed using the corresponding sense probe (Figure 2H).

\section{REFERENCES}

1.Danker, K., H. Hacke, J. Ramos, D. DeSimone, and D. Wedlich. 1993. V(+)-fibronectin expression and localization prior to gastrulation in Xenopus laevis embryos. Mech. Dev. 44:155-165.

2.David, R. and D. Wedlich. 2000. Xenopus cadherin-6 is expressed in he central and peripheral nervous system and in neurogenic placodes. Mech. Dev. 97:187-190.

3.Etard, C., D. Wedlich, A. Bauer, O. Huber, and M. Kuhl. 2000. Expression of Xenopus homologs of the beta-catenin binding protein pontin52. Mech. Dev. 94:219-222.

4.Hollemann, T. and T. Pieler. 1999. Xpitx-1: a homeobox gene expressed during pituitary and cement gland formation of Xenopus embryos. Mech. Dev. 88:249-252.

5.Hopwood, N.D., A. Pluck, and J.B. Gurdon. 1989. A Xenopus mRNA related to Drosophila twist is expressed in response to induction in the mesoderm and the neural crest. Cell 59:893-903.

6.Lee, J.E., S.M. Hollenberg, L. Snider, D.L. Turner, N. Lipnick, and H. Weintraub. 1995. Conversion of Xenopus ectoderm into neurons by NeuroD, a basic helix-loop-helix protein. Science 268:836-844.

7.Logel, J., D. Dill, and S. Leonard. 1992. Synthesis of cRNA probes from PCR-generated DNA. BioTechniques 13:604-610.

8.Schlosser, G. and R.G. Northcutt. 2000. Development of neurogenic placodes in Xenopus laevis. J. Comp. Neurol. 418:121-146.

9.Smith, J.C., B.M. Price, J.B. Green, D. Weigel, and B.G. Herrmann. 1991. Expression of a Xenopus homolog of Brachyury (T) is an immediate-early response to mesoderm induction. Cell 67:79-87.

We thank Benjamin Krauss and Bärbel Weber for excellent technical assistance. We are very grateful to Dr. Kris Henningfeld for critical reading of the manuscript. Address correspondence to Dr. Robert David, Abt. Biochemie der Universität Ulm, Albert-Einstein-Allee 11, D-89081 Ulm, Germany. email: robert.david@medizin.uni-ulm.de

Received 28 July 2000; accepted 30 October 2000.

\section{Robert David and Doris Wedlich \\ Universität Ulm \\ Ulm, Germany}

